\title{
SECOND DEGREE POLYNOMIALS AND THE FUNDAMENTAL THEOREMS OF HARMONIC ANALYSIS
}

\author{
KELLY MCKENNON \\ Department of Pure and Applied Mathematics, Washingtion State University \\ Pullman, Washington 99164 \\ (Received September 11, 1981)
}

ABSTRACT. The concept of a second degree polynomial with nonzero subdegree is investigated for Abelian groups, and it is shown how such polynomials can be exploited to produce elementary proofs for the Uniqueness Theorem and the Fourier Inversion Theorem in abstract harmonic analysis.

KEY WORDS AND PHRASES. Fourier inversion theorem, second degree polynomials. 1980 MATHEMATICS SUBJECT CLASSIFICATION CODE. 43A45, 12 A20.

\section{INTRODUCTION.}

Second degree polynomials. Let $G$ and $M$ be fixed, but arbitrary, Abelian topological groups. Let HOM(G,M) be the group of continuous homomorphisms of $G$ into $M$, equipped with the compact-open topology. Let $\operatorname{AFF}(G, M)$ be the set of all functions $G \rightarrow x \rightarrow b+f(x)$ such that $b \in M$ and $f \in \operatorname{HOM}(G, M)$. Write $S B H(G, M)$ for the set of continuous symmetric maps $\psi \mid G x G \rightarrow M$ such that, for each $a \in G$, the function $G \rightarrow x \rightarrow \psi(x, a)$ is in $\operatorname{HOM(G,M).~First~degree~polynomials~with~nonzero~subdegree~are,~by~definition,~}$ elements of $\operatorname{HOM}(G, M):$ i.e. continuous functions $f / G \rightarrow M$ such that

$$
f(x+y)-f(x)-f(y)=0 \text { for all } x, y \in G .
$$

Second degree polynomials with nonzero subdegree are defined to be continuous

$$
f(x+y+z)-f(x+y)-f(x+z)-f(y+z)+f(x)+f(y)+f(z)=0
$$

for all $x, y, z \in G$ - we write $P_{2}(G, M)$ for the set of all such.

The following observations are elementary:

$$
P_{2}(G, M) \text { is closed under (pointwise) addition; }
$$




$$
\begin{aligned}
& f(0)=0 \text { for all } f \in P_{2}(G, M) ; \\
& \operatorname{HOM}(G, M) \subset P_{2}(G, M) \text {. }
\end{aligned}
$$

For $f \mid G \rightarrow M$, define $f^{*} \mid G \rightarrow M$ by $f^{*}(x) \equiv f(-x)$ for all $x \in G$.

Clearly

$$
f^{*} \in P_{2}(G, M) \text { for all } f \in P_{2}(G, M) \text {. }
$$

A function $f \mid G \rightarrow M$ is called even if $f=f^{*}$. Write $E P_{2}(G)$ for the set of all even functions in $P_{2}(G)$. Inserting $x=y=-z$ in (2), we obtain with use of (4)

$$
3 f(x)-f(2 x)+f(-x)=0 .
$$

For $f \in P_{2}(G, M)$, (7) implies

$$
f \in E_{2}(G) \Leftrightarrow f(2 x)=4 f(x) \quad \text { for all } x \in G .
$$

If $f \in P_{2}(G, M)$ is even and $x=-z$ is put into (2), then

$$
2 f(x)+2 f(y)=f(x+y)+f(x-y) .
$$

Conversely, if (9) holds for $f \in P_{2}(G, M)$ inserting $x=y$ in (9) yields $f(2 x)=4 f(x)$. Thus (9) is equivalent to the evenness.

If $f \mid G \rightarrow M$ satisfies $f^{*}=-f$, it is said to be odd. Write $O P_{2}(G, M)$ for the set of all odd functions in $P_{2}(G, M)$. For $f \in P_{2}(G, M)$ (7) implies

$$
f \in O P_{2}(G, M) \Leftrightarrow f(2 x)=2 f(x) \text { for all } x \in G .
$$

Putting $x=2 t+y$ and $z=-2 t-y$ in (2), one obtains

$$
2 f(y)+f(2 t+y)+f(-2 t-y)-f(2 t+2 y)-f(-2 t)=0 .
$$

If $f$ is odd, (11) reduces to

$$
2[f(y)+f(t)-f(y+t)]=0 \text { for all } t, y \in G .
$$

Thus we have

$$
f \in \mathrm{OP}_{2}(G, M) \Rightarrow 2 f \in \operatorname{HOM}(G, M) .
$$

In view of $(10),(12)$, and (5), (13) can be improved in special cases:

$$
\begin{aligned}
& f \in O P_{2}(G, M) \Leftrightarrow f \in \operatorname{HOM}(G, M) \text { if either } \\
& (\forall x \in G)(\exists y \in G) \quad 2 y=x
\end{aligned}
$$

or

$$
\text { M has no element of order } 2 .
$$

In particular (14) holds when $G$ is compact and connected ([2] 24.25) or when $M$ is torsion-free. 
It is not true that (14) holds in general. Let $Z$ be the additive group of integers and $\mathrm{C}_{2}$ the cyclic group of order two with generator 1 . Define $\mathrm{p} \mid \mathrm{z} \oplus \mathrm{z} \rightarrow \mathrm{C}_{2}$ by

$$
p(m \mp n) \equiv \begin{cases}1 & \text { if both } m \text { and } n \text { are odd } \\ 0 & \text { otherwise }\end{cases}
$$

That $p$ is odd is clear. That $p$ is in $P_{2}\left(Z \boxplus z, C_{2}\right)$ is evident from the following. If $\mathbf{x}=\mathbf{m} \uplus \mathbf{n}$, if $\mathbf{y}=\mu \notin \mathcal{H}$ and $\mathbf{z}=\mathbf{M} \uplus \mathrm{N}$, then

\begin{tabular}{|c|c|c|c|c|c|c|c|}
\hline & $p(x+y+z)$ & $p(x)$ & $p(y)$ & $p(z)$ & $p(x+y)$ & $p(x+z)$ & $p(y+z)$ \\
\hline $\begin{array}{l}\mathrm{m}, \mathrm{n} \text { even } \\
\text { others arbitrary }\end{array}$ & $p(y+z)$ & 0 & $p(y)$ & $p(z)$ & $p(y)$ & $p(z)$ & $p(y+z)$ \\
\hline $\begin{array}{l}\text { m even } \\
\text { others odd } \\
\end{array}$ & 0 & 0 & 1 & 1 & 0 & 0 & 0 \\
\hline $\begin{array}{l}\text { m, } v \text { even } \\
\text { others odd }\end{array}$ & 0 & 0 & 0 & 1 & 1 & 0 & 0 \\
\hline $\begin{array}{l}\mathrm{m}, \mathrm{N} \text { even } \\
\text { others odd }\end{array}$ & 0 & 0 & 1 & 0 & 0 & 1 & 0 \\
\hline $\begin{array}{l}\mathrm{m}, \nu, \mathrm{N} \text { even } \\
\text { others odd }\end{array}$ & 0 & 0 & 0 & 0 & 1 & 1 & 0 \\
\hline $\begin{array}{l}\text { m, } \mu \text { even } \\
\text { others odd }\end{array}$ & 1 & 0 & 0 & 1 & 0 & 0 & 0 \\
\hline $\begin{array}{l}\text { m,M even } \\
\text { others odd }\end{array}$ & 1 & 0 & 1 & 0 & 0 & 0 & 0 \\
\hline $\begin{array}{l}m, \mu, v \text { even } \\
\text { others odd }\end{array}$ & 0 & 0 & 0 & 0 & 0 & 1 & 1 \\
\hline $\begin{array}{l}\mathrm{m}, \mu, \mathrm{N} \text { even } \\
\text { others odd } \\
\end{array}$ & 0 & 0 & 0 & 0 & 0 & 1 & 1 \\
\hline $\begin{array}{l}\mathrm{m}, \mu, \mathrm{N}, \nu \text { even } \\
\text { others odd }\end{array}$ & 1 & 0 & 0 & 0 & 0 & 1 & 0 \\
\hline $\begin{array}{l}\mathrm{m}, \mathrm{M}, \mathrm{V} \text { even } \\
\text { others odd } \\
\end{array}$ & 0 & 0 & 0 & 0 & 1 & 0 & 1 \\
\hline $\begin{array}{l}\mathrm{m}, \mathrm{M}, \mathrm{N} \text { even } \\
\text { others odd }\end{array}$ & 0 & 0 & 1 & 0 & 0 & 0 & 1 \\
\hline $\begin{array}{l}\mathrm{m}, \mathrm{M}, \mathrm{V}, \mathrm{N} \text { even } \\
\text { others odd }\end{array}$ & 1 & 0 & 0 & 0 & 0 & 1 & 0 \\
\hline $\begin{array}{l}\mathrm{m}, \mu, \mathrm{M} \text { even } \\
\text { others arbitrary }\end{array}$ & 0 & 0 & 0 & 0 & 0 & 0 & 0 \\
\hline
\end{tabular}

The cases in which $\mathbf{n}$ is even can be found from the above table invoking the symmetry of $P$.

Thus

$$
p \in O P_{2}\left(Z \oplus Z, C_{2}\right) \text { and } p \in \operatorname{HOM}\left(Z \oplus Z, C_{2}\right) \text {. }
$$

If $\psi$ is in $\operatorname{SBH}(G, M)$ and $f(x)=\psi(x, x)$ for all $x \in G$, then $f$ is said to be quadratic - the set of all such will be written $Q D(G, M)$. That 


$$
\mathrm{QD}(\mathrm{G}, \mathrm{M}) \subset \mathrm{EP}_{2}(\mathrm{G}, \mathrm{M})
$$

is elementary. On the other hand, consider the function $p$ defined in (15) and assume that $p(x)=\psi(x, x)$ for all $x \in z \oplus z$. Then

$$
\begin{aligned}
1 & =(p(1 \oplus 1)=\psi(1 \oplus 0,1 \oplus 0)+\psi(1 \oplus 0,0 \oplus 1)+\psi(0 \oplus 1,1 \oplus 0)+\psi(0 \oplus 1,0 \oplus 1) \\
& =p(1 \oplus 0)+2 \psi(1 \oplus 0,0 \oplus 1)+p(0 \oplus 1)=0+0+0=0,
\end{aligned}
$$

an absurdity. Thus

$$
p \in E P_{2}\left(Z \oplus Z, G_{2}\right) \text { but } p \in Q D(G, M) .
$$

For $\psi \in \operatorname{SBH}(G, M)$ and $f(x)=\psi(x, x)$, the equation $f(x+y)=f(x)+f(y)+2 \psi(x, y)$ shows that

$$
f \in \operatorname{HOM}(G, M) \Leftrightarrow(\forall x, y \in G) \quad 2 \psi(x, y)=0 .
$$

There is a partial converse to (17). If $h$ is any element of $P_{2}(G, M)$, then

$$
h^{\#} \mid G x G \rightarrow(x, y) \rightarrow h(x+y)-h(x)-h(y) \text { is in } \operatorname{SBH}(G, M)
$$

as follows readily from (2). If $h$ is even and $f=2 h$, a direct computation with

(8) yields $f(x)=h^{\#}(x, x)$ for all $x \in G$. Thus

$$
\left\{2 h: h \in E P_{2}(G, M)\right\} \subset Q D(G, M) \text {. }
$$

Any function $f \in P_{2}(G, M)$ of the form $2 h$ for $h \in P_{2}(G, M)$ can be written $f=\left(h+h^{*}\right)+\left(h-h^{*}\right)$ and so

$$
2 h \in \mathrm{EP}_{2}(G)+0 P_{2}(G) \text {. }
$$

\section{TRANSLATION-PSEUDO-INVARIANCE.}

For $f \mid G \rightarrow M$ and $a \in G$, the translation $f_{a} \mid G \rightarrow M$ is defined by $f_{a}(x)=f(x-a)$ for all $x \in G$. Thus the only translation-invariant functions are constants. A function $f \mid G \rightarrow M$ will be said to be translation-pseudo-invariant (TPI) if, for each $a \in G$, there exists $\alpha^{f, a} \in \operatorname{AFF}(G, M)$ such that

$$
f_{a}=\alpha^{f, a}+f .
$$

THEOREM 1. The TPI-functions $f$ such that $f(0)=0$ are the elements of $P_{2}(G, M)$.

PROOF. Let $f \in P_{2}(G, M)$ be arbitrary. Define $\psi \mid G \times G \rightarrow M$ by $\psi(x, y) \equiv f(x+y)-f(x)-f(y)$ for all $x, y \in G$. Direct computation with (2) shows that $\psi$ is in $\operatorname{SBH}(G, M)$. Thus, for all $a, x \in G$,

$$
f_{a}(x)=f(x-a)=\psi(x,-a)+f(x)+f(-a)
$$


so

$$
f_{a}=\alpha^{f, a}+f \text { where } \alpha^{f, a} \mid G \rightarrow x \rightarrow f(-a)+\psi(x,-a) .
$$

Now let $f$ be an arbitrary TPI-function. Then, for each $a \in G$, there exists $g(a) \in M$ and $\eta^{a} \in \operatorname{HOM}(G, M)$ such that

$$
f_{a}(x)=g(a)+\eta^{a}(x)+f(x) \quad \text { for all } x \in G .
$$

For $a, b, x \in G$ we have

$$
\begin{gathered}
g(a+b)+\eta^{a+b}(x)+f(x)=f_{a+b}(x)=f_{a}(-b+x) \\
g(a)+\eta^{a}(-b+x)+f(-b+x)=g(a)+\eta^{a}(-b)+\eta^{a}(x)+g(b)+\eta^{b}(x)+f(x),
\end{gathered}
$$

whence follows that

$$
g(a+b)+n^{a+b}=g(a)+g(b)+n^{a}(b)+n^{a}+n^{b} .
$$

Since the left side of (24) is symmetric in a and b, the right side is as well. Thus

$$
n^{a}(b)=n^{b}(a) \quad \text { for } a l l a, b \in G \text {. }
$$

Consequently the function $\psi \mid G \times G \rightarrow(x, y) \rightarrow \eta^{x}(y)$ is symmetric and is, in fact, in $\operatorname{SBH}(G, M)$. Hence $\eta^{a+b}=n^{a}+n^{b}$ so (24) becomes

$$
g(a+b)=g(a)+g(b)+\psi(a, b) \quad \text { for } a 11 a, b \in G \text {. }
$$

For $x, y, z \in G$, the above yields

$$
\begin{gathered}
g(x+y+z)-g(x+y)-g(x+z)-g(y+z)+g(x)+g(y)+g(z)= \\
{[g(x+y+z)-g(x+y)-g(z)]-[g(x+z)-g(x)-g(z)]-[g(y+z)-g(y)-g(z)]=} \\
\psi(x+y, z)-\psi(x, z)-\psi(y, z)=0
\end{gathered}
$$

so $g \in P_{2}(G, M)$. Furthermore, for each $x \in G$,

$$
f(x)=f_{-x}(0)=g(-x)+n^{-x}(0)+f(0)=g^{\star}(x)
$$

\section{THE FUNDAMENTAL THEOREMS OF ABELIAN ABSTRACT HARMONIC ANALYSIS.}

In the sequel $\mathrm{G}$ will be a fixed, but arbitrary, locally compact, Abelian, Hausdorff, topological group (LCA gp.) For any such group G we write $\lambda_{G}$ for a Haar measure on $G, L^{p}(G)$ for the $L^{p}$-space relative to $\lambda_{G}(p \in[1, \infty])$, and $\mathbb{P}(G)$ for the Banach space of bounded, complex, regular Borel measures on $G$ and $B(G)$ for the set of continuous, positive-definite functions on $G$. Let $T$ be the group of complex numbers of unit modulus and \& any LCA group topologically isomorphic to $\operatorname{HOM}(G, T)$. 
The fundamental theorems of Abelian abstract harmonic analysis are as follows:

(DUALITҰ THEOREM) There exists a continuous bihomomorphism $\bullet$ from

$G \times$ \& to $T$ such that, if for $a \in G$ and $b \in \&$ the $\bar{a}$ and $\bar{b}$ are defined by

$$
\bar{a} \mid \& \exists y \rightarrow a \bullet y \text { and } \bar{b} \mid G \ni x \rightarrow x \bullet b \text {, }
$$

then the maps

$$
\mathrm{G} \ni \mathrm{a} \rightarrow \overline{\mathrm{a}} \epsilon \operatorname{HOM}(\&, \mathrm{~T}) \text { and } \& \ni \mathrm{b} \rightarrow \overline{\mathrm{b}} \in \operatorname{HOM}(G, T)
$$

are both topological isomorphisms;

(UNIQUENESS THEOREM) if, for each $\mu \in \mathbb{R}(G), \hat{\mu}$ is defined by

$$
\hat{\mu} \mid \& \ni y \rightarrow \int_{G}-x \cdot y d \mu(y) \text {, }
$$

then $\hat{\mu}$ is nonzero whenever $\mu$ is nonzero;

(INVERSION THEOREM) if, for each $f \in L^{1}(G)$ and $h \in L^{1}(\&), \hat{f}$ and $\check{h}$ are defined by

$$
\hat{\mathrm{f}} \mid \& \ni \mathrm{y} \rightarrow \int_{\mathrm{G}} \mathrm{f}(\mathrm{t})-\mathrm{t} \odot \mathrm{y} \mathrm{d} \lambda_{\mathrm{G}}(\mathrm{y}) \text { and } \check{\mathrm{h}} \mid \mathrm{G} \ni \mathrm{x} \rightarrow \int_{\mathcal{g}} \mathrm{h}(\mathrm{s}) \mathrm{x} \in \mathrm{s} \mathrm{d} \lambda,(s) \text {, }
$$

then $\lambda_{\&}$ can be normalized such that, whenever $\hat{\mathbf{f}}$ is in $L^{1}(\&)$ for $f \in L^{1}(G)$, then $\hat{\mathbf{f}}^{\vee}=\mathbf{f} ;$

(PLANCHEREL'S THEOREM) $\lambda_{\&}$ can be normalized such that, for each $f \in L^{2}(G) \cap L^{1}(G)$,

$$
\int_{G}|\mathrm{f}|^{2} \mathrm{~d} \lambda_{G}=\int_{\&}|\hat{\mathrm{f}}|^{2} \mathrm{~d} \lambda_{\&} ;
$$

(BOCHNER'S THEOREM) $\quad \mathbb{P}(G)=\left\{\hat{\mu}: \mu \in \mathbb{M}(\&)^{+}\right\}$.

There are two basic approaches to the proof of the fundamental theorems. The more modern approach is based on the Gelfand-Naimark theory of commutative Banach Algebras. It is based on the observation that, under the convolution operation * defined by

$$
f * h(x)=\int_{G} f(x-t) h(t) d t \quad \text { for all } f, h \in L^{1}(G) \text { and } x \in G \text {, }
$$

$L^{1}(G)$ is a commutative Banach algebra whose spectrum is topologically isomorphic to HOM(G,T). This approach was taken by D.A. Raikov in [5]. Here Bochner's Theorem (29) is established first, and the other theorems deduced from it (see [1] and [6] for more recent accounts).

The original approach was based on the structure theory of LCA groups as developed by L.S. Pontryagin and E.R. van Kampen. Theorem (25) in full generality 
was first published in 1935 by van Kampen [7]. The other fundamental theorems are then deduced from (25) by judicious use of the Stone-Weierstrass Theorem and somewhat delicate construction of approximate identities for $L^{1}(G)$ based on the structure theory (see [2] and [3] for instance).

Our approach here is based on the structure theory and takes (25) for granted. It however is not dependent on the Stone-Weierstrass Theorem and the approximate identity exploited is relatively simple. The basic techniques are use of TPIfunctions and simple complex analysis. The theorems (27), (28) and (29) are essentially equivalent in the sense that, once one of them is established, the task of establishing the others is relatively simple. Consequently we shall employ our method to prove only (26) and (27).

Let $T^{\mathbb{C}}$ be the multiplicative group of nonzero complex numbers. We shall make exception to our previously adopted convention by employing multiplicative (rather than additive) notation for $T^{\mathbb{C}}$ and its subgroup $T$. We define the complexification $\mathrm{G}^{\mathbb{C}}$ of $\mathrm{G}$ to be an abstract group isomorphic to $\operatorname{HOM}\left(\&, \mathrm{~T}^{\mathbb{C}}\right)$ containing $\mathrm{G}$ as a subgroup and extend - to a map on $G^{\mathbb{C}} \oplus$ \& such that, if for each $a \in G^{\mathbb{C}}$, $\bar{a}$ is defined by $\bar{a} \mid \& \ni y \rightarrow a \otimes y$, then

$$
G^{C} \rightarrow a \rightarrow \bar{a} \in \operatorname{HOM}\left(G, T^{\mathbb{C}}\right) \text { is an isomorphism. }
$$

In the special case $G=T$, we have $\&=Z$ and

$$
z \circ n=z^{n} \quad \text { for all } z \in T^{\mathbb{C}} \text { and } n \in Z \text {. }
$$

As a second example, consider finite dimensional real Hilbert space E. Then $\mathrm{G}=\mathscr{H}=\mathrm{E}$ and $\mathrm{E}^{\mathbb{C}}$ can be taken any complex Hilbert space in which $\mathrm{E}$ is a real form-here

$$
\alpha \ominus \beta=\mathrm{e}^{-i\langle\alpha, \beta\rangle} \quad \text { for all } \alpha \in \mathrm{E}^{\mathbb{6}}, \beta \in \&=\mathrm{E} \text {. }
$$

A function $f$ which is the restriction to $G$ of a function $f^{\mathbb{C}} \mid G^{\mathbb{C}} \rightarrow \mathbb{G}$, such that

$$
\mathbf{f}^{\mathfrak{C}} \circ \sigma \text { is analytic for each } \sigma \in \operatorname{AFF}\left(C, G^{\mathbb{C}}\right) \text {, }
$$

is said to be entire--the Uniqueness Theorem for analytic functions implies that the function $f^{\mathbb{C}}$ is unique. For $f$ entire and $a \in G^{\mathbb{C}}$, we define the translation $f_{a}$ of f by

$$
\mathbf{f}_{\mathbf{a}} \mid \mathrm{G} \rightarrow \mathrm{x} \quad \mathbf{f}^{\mathbb{C}}(\mathbf{x}-\mathbf{a})
$$


The complexification $\&^{\mathbb{C}}$, the concept of an entire function defined on $\&^{\mathbb{C}}$, and its translations are defined analogously.

We write $L_{\varepsilon}^{1}(G)$ for the set of all entire functions $f$ on $G$ such that, for each $\sigma \in \operatorname{AFF}\left(\mathbb{C}, G^{\mathbb{C}}\right)$ and $\delta>0$,

$$
\sup \left\{\left\|f_{\sigma(z)}\right\|_{1}:|z| \leq \delta\right\}<\infty
$$

(where \|\| , is the $L^{1}$-norm). In particular, $L_{\varepsilon}^{1}(G) \subset L^{1}(G)$.

LEMMA 1. Let $f \in L_{\varepsilon}^{1}(G)$ and $a \in G^{\mathbb{C}}$ be arbitrary. Then

$$
\int_{G} f_{a} d \lambda_{G}=\int_{G} \mathbf{f} d \lambda_{G}
$$

PROOF. The function $\bar{a} \mid \& \ni y \rightarrow a$ y can be written $|\bar{a}| \cdot \frac{\bar{a}}{|\bar{a}|}$ where $|\bar{a}| \in \operatorname{HOM}\left(\&, \mathbb{R}^{+}\right)$and $\frac{\bar{a}}{|\bar{a}|} \in \operatorname{HOM}(\&, T)$. Define $\sigma \in \operatorname{HOM}\left(\mathbb{C}, G^{\mathbb{b}}\right)$ by letting, for each $z \in \mathbb{C}, \sigma(z)$ be the element of $G^{\mathbb{C}}$ such that

$$
\overline{\sigma(z)} \mid \& \ni y \rightarrow \exp (z \ln |\bar{a}(y)|)
$$

Let $c \in G$ be such that $\bar{c}=\frac{\bar{a}}{|\bar{a}|}$ and define $\tau \in \operatorname{AFF}\left(\mathbb{C}, G^{\mathbb{C}}\right)$ by

$$
\tau(z)=c+\sigma(z) \quad \text { for } a 11 \quad z \in \mathbb{C} \text {. }
$$

Then (35) implies that, for any simple closed contour $\gamma$ in $\mathbb{C}$,

$$
\int_{\gamma}|f(x-\tau(z))| d \lambda_{G}(x) d z<\infty
$$

so it follows from Fubini's Theorem and Cauchy's Theorem that

$$
\int_{\gamma} \int_{G} f(x-\tau(z)) d \lambda_{G}(x) d z=\int_{G} \int_{\gamma} f(x-\tau(z)) d z d \lambda_{G}(x)=\int_{G} 0 d \lambda_{G}=0
$$

Morera's Theorem now implies that the function $F \mid c \ni z \rightarrow \int_{G} f(x-\tau(z)) d \lambda_{G}(x)$ is analytic. For imaginary $z, \overline{\sigma(z)}$ is in $\operatorname{HOM}(\xi, T)$ so $\sigma(z)$ is in G--hence $\tau(z)$ is in $\mathrm{G}$ as we11 and we have

$$
F(z)=\int_{G} f_{\tau(z)} d \lambda_{G}=\int_{G} f d \lambda_{G}
$$

by the invariance of the Haar integral. The Uniqueness Theorem for analytic functions now implies that (37) holds for all $z \in \mathbb{C}$. In particular $F(0)=\int_{G} f d \lambda_{G} \cdot$ But $\tau(0)=a$, so (36) holds. Q.E.D.

Relative to study of the Fourier Transformation, the utility of $P_{2}\left(G, T^{\mathbb{C}}\right)$ is dependent on the size of $P_{2}\left(G, T^{C}\right) \cap L^{1}(G)$. This size can vary, as is shown by 
Theorems 2 and 3 below.

LEMMA 2. Let $\mathrm{E}$ be finite dimensional real Hilbert space and $k \in] 0,1[$. Define $\mathrm{f} \mid \mathrm{E} \rightarrow \boldsymbol{c}$ by

$$
f(x) \equiv k^{<x, x>} \text { for all } x \in E
$$

Then $\mathrm{f}$ is in $\mathrm{EP}_{2}\left(\mathrm{E}, \mathrm{T}^{\mathbb{C}}\right) \cap \mathrm{L}_{\varepsilon}^{1}(\mathrm{E})$ and

$$
\hat{f}(x)=k^{<\frac{x}{\ln k}, \frac{x}{\ln k}>/ 4} \cdot\|f\|_{1} \text { for all } x \in E
$$

(where $\mathrm{E}$ is the dual of itself as in (33)).

PROOF. That $f$ is in $\mathrm{EP}_{2}$ (E) follows from (17).

Let $E^{\mathbb{C}}$ and $\odot$ be as in (33) and let ${ }^{\circ}$ be the conjugation of the Hilbert space $E^{\mathbb{C}}$ leaving the elements of $E$ fixed. Then the function $f^{\mathbb{C}} \mid E^{\mathbb{C}} \rightarrow z \rightarrow k^{\left\langle z, z^{\circ}\right\rangle}$ is analytic so $f$ is entire. Lemma 1 implies that, for each $y \in E$, $\|f\|_{1}=\int_{E} k^{\left\langle t,-i y,(t, i y)^{\circ}\right\rangle} d \lambda_{E}(t)=\int_{E} k^{\langle t, t\rangle} e^{-2 i<1 n k \cdot y, t\rangle} k^{-\langle y, y\rangle} d \lambda_{E}(t)$. Letting $x=2 \ln k \cdot y$, we have (39). Q.E.D.

LEMMA 3. Let $K$ be a compact subgroup of $G$ and $h$ an element of $P_{2}\left(G^{\mathbb{C}}, T^{\mathbb{C}}\right)$. Then $h(K) \subset T$.

PROOF. It will suffice to show that $h^{2}(K) \subset T$ (where $\left.h^{2} \mid G^{\mathbb{C}} \ni x \rightarrow h(x)^{2}\right)$. In view of $(21)$, there exist $p \in E P_{2}\left(G^{\mathbb{C}}, T^{\mathbb{C}}\right)$ and $g \in O P_{2}\left(G^{\mathbb{C}}, T^{\mathbb{C}}\right)$ with $h^{2}=p \cdot g$.

From (13) we have $g^{2} \in \operatorname{HOM}\left(G^{\mathbb{C}}, T^{\mathbb{C}}\right)$, whence follows that $g(K)^{2}$ is a compact subgroup of $\mathrm{T}^{\mathbb{6}}$. Thus $\mathrm{g}(\mathrm{K}) \subset \mathrm{T}$.

Let $\mathrm{p}^{\#} \mid \mathrm{G}^{\mathbb{C}} \times \mathrm{G}^{\mathbb{\mathfrak { C }}} \rightarrow \mathrm{T}^{\mathbb{\complement}}$ be defined by

$$
p^{\#}(x, y) \equiv \frac{p(x+y)}{p(x) p(y)} \quad \text { for } x, y \in G^{\mathbb{C}} \text {. }
$$

From (20) we have that $\mathrm{p}^{\#} \in \operatorname{SBH}\left(G^{\mathbb{C}}, \mathrm{T}^{\mathbb{C}}\right)$. Thus, for each $x \in G, \mathrm{p}^{\sharp}(x, K)$ is a compact subgroup of $T^{\mathbb{C}}$ and so a subgroup of $T$. Furthermore, by (8),

$$
\mathrm{p}^{\#}(\mathrm{x}, \mathrm{x})=\frac{\mathrm{p}(2 \mathrm{x})}{\mathrm{p}(\mathrm{x}) \cdot \mathrm{p}(\mathrm{x})}=\mathrm{p}(\mathrm{x})^{2}
$$

whence follows that $p(K) \subset T$.

Final1y,

$$
h^{2}(k) \subset p(K) \cdot g(K) \subset T \cdot T=T . \quad \text { Q.E.D. }
$$


THEOREM 2. There are LCA groups $G$ such that $L^{1}(G) \cap P_{2}\left(G^{\mathbb{C}}, T^{\mathbb{d}}\right)$ is void.

PROOF. Let $G$ be an LCA group with a sequence $S_{n}$ of compact subgroups such that $\lim _{n \rightarrow \infty} \lambda_{G}\left(S_{n}\right)=\infty$ (such as a discrete group which contradicts Burnside's conjecture). Then, for $h \in P_{2}\left(G^{\mathbb{C}}, T^{\mathbb{Q}}\right)$, Lemma 3 implies that $h\left(S_{n}\right) \subset T$ for each $n$. Thus

$$
\|\mathrm{h}\|_{1} \geq \lim _{\mathbf{n}} \int_{S_{n}}|\mathrm{~h}| \mathrm{d} \lambda_{G}=\lim _{\mathbf{n}} \lambda_{G}\left(S_{n}\right)=\infty . \quad \text { Q.E.D. }
$$

LEMMA 4. Suppose that $G$ is compact and that $f$ is a linear combination of characters. Then $\hat{\mathrm{f}}^{\curvearrowright}=\lambda_{G}(G) \cdot f$.

PROOF. Evidently we need only consider the case $f=\bar{b}$ for $b \in \&$. Since the fact that $(\bar{b})_{a}=\bar{b}(-a) \cdot \bar{b}$ implies $\bar{b}(-a) \cdot \int_{G} \bar{b} d \lambda_{G}=\int_{G}(\bar{b})_{a} d \lambda_{G}=\int_{G} \bar{b} d \lambda_{G}$ for each $a \in G$, it follows that

$$
\int_{G} \bar{b} d \lambda_{G}=\left\{\begin{array}{cc}
0 & \text { if } b \neq 0 \\
\lambda(G) & \text { if } b=0
\end{array}\right. \text {. }
$$

Consequently $\bar{b}^{\wedge}$ is $\lambda_{G}(G)$ times the characteristic function of the singleton $b$. Similarly $\bar{b}^{\wedge \nu}$ must be $\lambda_{G}(G) \cdot \bar{b}$. Q.E.D.

Let $\Re$ be the directed set of open neighborhoods of 0 in $G$.

THEOREM 3. There is an open subgroup $S$ of $G$, a set $G \subset L^{1}(G)$ and a surjection $\mathfrak{N} \times \mathbb{N} \ni(A, m) \rightarrow f^{(A, m)} \in G$ satisfying the following properties.

$\left.f\right|_{G \backslash S}=0$ and $\left.f\right|_{S}$ is in the convex hull of $P_{2}\left(S, T^{c}\right)$ for all $f \in G$;

$f(0)=1$ and $1 \geq f \geq 0$ for all $f \in G$;

For each $A \in \mathfrak{N}$, there exists $B \in \mathfrak{N}$ such that $B \subset A$ and

$$
\lim _{m \rightarrow \infty} \int_{B} f^{(A, m)} d \lambda_{G} / \int_{G \backslash B} f^{(A, m)} d \lambda_{G}=\infty ;
$$

there exists a net $f_{\alpha}$ in $G$ such that the net $\hat{f}_{\alpha} /\left\|f_{\alpha}\right\|_{1}$ converges uniformly to 1 on compacta;

$\hat{\mathrm{f}} \in \mathrm{L}_{\varepsilon}^{1}(\xi)$ for each $\mathrm{f} \in \mathrm{G}$;

$\hat{\mathbf{f}}^{\vee}(0)=\hat{h}^{2}(0)$ for all $\mathrm{f}, \mathrm{h} \in \mathrm{G}$.

PBOOF. The Pontryagin-van Kampen Structure Theorem ([2] 24.8) implies that G may be regarded as the direct sum of $n$-dimensional real Hilbert space $E$ and a subgroup $H$ containing a compact, open subgroup $M$. We denote $E \oplus M$ by $S$ and its dual 
group by 8 . The Duality Theory ([2] $24.11,24.10$ ) implies that the map $\sigma \mid \& \rightarrow S$, determined by the equation

$$
\overline{\sigma(y)}=\left.\bar{y}\right|_{S} \quad \text { for } \text { all } y \in \&
$$

is a topological epimorphism, that $e \equiv\left\{y \in \cdot S:\left.\bar{y}\right|_{M}=1\right\}$ may be identified with the dual of $E$, and that $m \equiv\left\{y \in g:\left.\bar{y}\right|_{E}=1\right\}$ may be identified with the dual of $M$.

The map $\sigma \mid \& \rightarrow g$ has a canonical extension (also denoted by $\sigma$ ) to an epimorphism of $G^{\mathbb{C}}$ onto $g^{\mathbb{C}}$ such that (46) holds for all $\mathrm{y} \in \mathcal{S}^{\mathbb{C}}$. Since $M$ is compact and continuous homomorphisms preserve compact groups, it follows that $m=m^{C}$ so we can (and shall) identify $e^{\mathbb{C}} \oplus \pi$ with $\mathrm{s}^{\boldsymbol{c}}$.

As in (33) we may view $\mathrm{E}^{\mathfrak{C}}$ as a complex Hilbert space in which $\mathrm{E}$ is a real form. Let ${ }^{\circ}$ be the conjugation of $E^{C}$ leaving the elements of $E$ fixed. Since $e$ is topologically isomorphic to $E$, there exists an isomorphism $\theta \mid E^{\complement} \rightarrow e^{\mathbb{C}}$ sending $E$ to satisfying

$$
x \bullet \theta(y)=e^{-i<x, y>} \text { for all } x \in E \text { and } y \in E^{\mathbb{C}}
$$

Homomorphisms $n \in \operatorname{HOM}\left(\&^{\mathbb{C}}, E^{\mathbb{C}}\right)$ and $\mu \in \operatorname{HOM}\left(\&^{\mathbb{C}}, m\right)$ are determined by the equation

$$
\sigma(y)=\theta \circ n(y) \oplus \mu(y) \quad \text { for all } y \in \mathscr{S}^{\mathbb{C}} \text {. }
$$

We may (and shal1) assume that $\lambda_{S}$ is the restriction to $S$ of $\lambda_{G}$, that

$$
\begin{aligned}
& \lambda_{M}(M)=1=\lambda_{m}(m) \text {, that } \lambda_{E} \text { satisfies } \\
& \qquad \int_{S} g d \lambda_{S}=\int_{E} \int_{M} g(x+y) d \lambda_{M}(y) d \lambda_{E}(x) \text { for a11 } g \in L^{1}(S),
\end{aligned}
$$

that $\left.\theta\right|_{E}$ is $\lambda_{E}-\lambda_{E}$ - measure preserving, that $\lambda_{g}$ satisfies

$$
\int_{\delta} g d \lambda_{g}=\int_{e} f_{m} g(x+y) d \lambda_{m}(y) d \lambda_{e}(x) \text { for all } g \in L^{1}(g)
$$

Weil's Theorem ([3] 28.54) permits us to normalize $\lambda_{\&}$ such that

$$
\int_{g} g d \lambda_{g}=\oint_{g} g \circ \sigma d \lambda_{\&} \text { for each } g \in L^{1}(g) \text {. }
$$

For each $A \in \Re$, let $A_{E}$ and $A_{M}$ be the canonical projections (relative to the direct sum $S=E(\rightarrow M)$ of $A$ into $E$ and $M$ respectively. Since to each $x \in M \backslash A_{M}$ there corresponds at least one $y \in S$ such that $\bar{x}(y)=\bar{y}(x) \neq 1$, it follows from the compactness of $M \backslash A_{M}$ there is a finite subset $F(A)$ of $s$ such that $M \backslash A_{M} \subset\{x \in M: x \otimes y \neq 1$ for some $y \in F(A)\}$. Define 


$$
h_{A} \equiv \frac{1}{4}\left[1+\prod_{y \in F(A)} \frac{\bar{y}+\overline{(-y)}}{2}\right]^{2} .
$$

Then $h_{A}$ satisfies

$$
h_{A}(0)=1,1 \geq h_{A} \geq 0 \text {, and } h_{A}(x)<1 \text { for all } x \in M \backslash A_{M} \text {. }
$$

For each $m \in \mathbb{N}$, define $f^{(A, m)} \mid G \rightarrow \mathbb{R}^{+}$by

$$
\left.f^{(A, M)}\right|_{G \backslash S} \equiv 0 \text { and } f^{(A, m)}(x+y)=e^{-m<x, x>} h_{A}(y) \text { for } x \in E, y \in M \text {. }
$$

That (40) holds is evident from Lemma 2, that $\operatorname{HOM}(S, T) \subset P_{2}(S, T)$, and the construction of $f^{(A, m)}$. Let $G$ be the set of all such functions $f^{(A, m)}$. That (41) holds is obvious.

For each $r>0$ let $E(r)$ be the set $\{x \in E:\langle x, x\rangle \leq r\}$. Then, for each $m \in \mathbb{N}$, the change of variables $s=\sqrt{m} \times$ yields

$$
\underset{E(r)}{\int} e^{-m<x, x>} d \lambda_{E}(x)=m^{-n / 2} \int_{E(m r)} e^{-\langle s, s>} d \lambda_{E}(s) .
$$

Let $D$ be an open subset of $\left\{x \in M: h_{A}(x)>\frac{1}{2}\right\}$ and $r^{\prime}$ a positive number such that $B \equiv E\left(r^{\prime}\right)+D$ is a subset of $A$. Then (54) yields

$$
\begin{aligned}
& \lim _{m \rightarrow \infty} \frac{\int_{B} f^{(A, m)} d \lambda_{G}}{\int_{G \backslash B} f^{(A, m)} d \lambda_{G}} \geq \lim _{m \rightarrow \infty} \frac{\frac{1}{2 / 2} \int_{E\left(r^{\prime}\right)} e^{-m<x, x>} d \lambda_{E}(x)}{E \backslash E\left(r^{\prime}\right)} e^{e^{-m<x, x>} d \lambda_{E}(x)}
\end{aligned}
$$

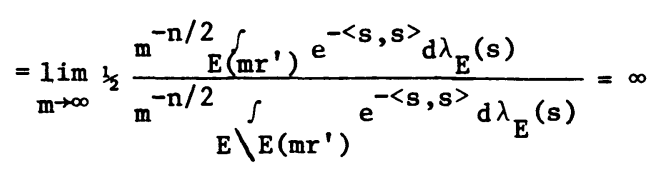

which proves (42).

For $A \in \mathfrak{N}$ and $r>0$, let $A_{r}^{2} \equiv\{y \in s:|x \bullet y-1|<r$ for all $x \in A\}$.

Since ном $(\&, T)$ bears the compact-open topology, $\left\{A_{r}^{+}: A \in R, r>0\right\}$ is a fundamental family of compact subsets of \& Let $A \in \mathfrak{N}$ and $r \in] 0,1[$ be arbitrary. For $\delta>0$, apply (42) to obtain $B \in \mathfrak{N}$ and $f \in Q$ such that $B \subset A$ and

$$
\int_{B} f d \lambda_{G} \geq \frac{1-r}{r} \int_{G \backslash B} f d \lambda_{G}
$$


so that

$$
\frac{\int_{G} f d \lambda_{G}}{\int_{G} f d \lambda_{G}} \leq r .
$$

Thus, for each $y \in A_{r}^{\perp}$,

$$
\begin{gathered}
\left|\frac{\hat{f}(y)}{\|f\|_{1}}-1\right| \leq\left|\frac{\int_{B} f \cdot(1-\bar{y}) d \lambda_{G}}{\|f\|_{1}}\right|+\left|\frac{G \int_{B} f \cdot(1-\bar{y}) d \lambda_{G}}{\|f\|_{1}}\right| \leq \\
\quad \\
\quad \frac{\|f\|_{1}}{\|f\|_{1}}+\frac{2 \cdot \int_{G \backslash B}|f| d \lambda_{G}}{\|f\|_{1}} \leq 3 r .
\end{gathered}
$$

This proves 43 .

Let $f=f^{(A, m)}$ be an arbitrary element of $G$. From (40) and (46) follows that for each $y \in \&$,

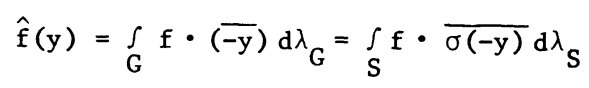

so that by (49) and (47)

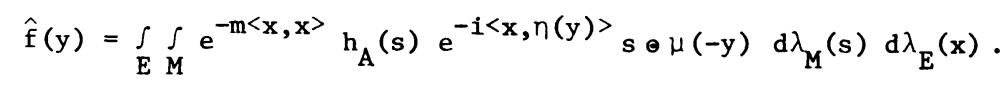

From (39), (54) and (55) follows, upon setting $c \equiv \int_{E} e^{-\langle x, x\rangle} d \lambda_{E}(x)$,

$$
\hat{f}(y)=e^{-\frac{1}{4 m}\langle n(y), \eta(y)\rangle} \cdot c \cdot m^{-n / 2} \cdot\left(\left.h_{A}\right|_{M}\right)^{\wedge}(\mu(y)) \text { for all y } \in \text { \&. }
$$

Since $\delta^{\mathbb{C}}=\mathbb{e}^{\mathbb{C}} \mathbb{\oplus} \boldsymbol{m}$, the function

$$
\&^{\mathbb{C}} \rightarrow y \rightarrow e^{-\frac{1}{4 m}\left\langle n(y), n(y)^{\circ}\right\rangle} \cdot(4 m)^{-n / 2} \cdot\left(\left.h_{A}\right|_{M}\right)^{\wedge}(\mu(y))
$$

is analytic--as an extension of $\hat{\mathbf{f}}$, it will be denoted $\hat{\mathbf{f}}^{\mathbf{C}}$.

An element $b$ of $\&^{c}$ can be written uniquely as $u+v$ where $u \in \&$ and $v \in \&^{\mathbb{C}}$ satisfies $\bar{v}=|\bar{b}|$. For such $b=u+v$ and $y \in \&$,

$$
\left|e^{-\frac{1}{4 m}\left\langle n(y-b), n(y-b)^{\circ}\right\rangle}\right|=e^{-\frac{1}{4 m}\langle n(y-u), n(y-u)\rangle} e^{\frac{1}{4 m}\langle n(v), n(v)\rangle}
$$

Since the support of $\hat{h}_{A}$ is a subset of a finite set $F(A) \cup(-F(A))+F(A) \cup(-F(A))$, the number $w(A) \equiv \int_{m}\left|\left(\left.h_{A}\right|_{M}\right)^{\wedge}\right| d \lambda_{m}$ is finite. Thus, application of (51), (50), (57), and (54) yields 


$$
\begin{aligned}
& \left\|(\hat{\mathrm{f}})_{b}\right\|_{1}=\left\{\left|\hat{f}^{\mathbf{c}}(y-b)\right| d \lambda_{g}=\int_{e}\left|e^{-\frac{1}{4 m}\langle n(y-b), n(y-b)\rangle}\right| d \lambda_{e}(y) \cdot(4 m)^{-n / 2} \cdot w(A)=\right. \\
& \int^{-\frac{1}{4 m}\langle n(y-u), n(y-u)\rangle} d \lambda_{e}(y) \cdot e^{\frac{1}{4 m}\langle n(v), n(v)\rangle} \cdot c \cdot(4 m)^{-n / 2} w(A)= \\
& \int_{E} e^{-\frac{1}{4 m}\langle x, x>} d \lambda_{E}(x) \cdot e^{\frac{1}{4 m}\langle n(v), n(v)\rangle} \cdot c \cdot(4 m)^{-n / 2} \cdot w(A)=e^{\frac{1}{4 m}\langle n(v), n(v)\rangle} \cdot c^{2} \cdot w(A)
\end{aligned}
$$

This proves (44).

From Lemma 4 follows that

$$
\int_{m}\left(\left.\mathrm{~h}_{\mathrm{A}}\right|_{M}\right)^{\wedge} \mathrm{d} \lambda_{m}=\left(\left.\mathrm{h}_{\mathrm{A}}\right|_{M}\right)^{\wedge \sim}(0)=1 .
$$

Consequently (56), (49), and (54) yield

$$
\begin{gathered}
\hat{\mathrm{f}}^{\sim}(0)=\int_{\&} \hat{\mathrm{f}} \mathrm{d} \lambda_{\&}=\int_{E} e^{-\frac{1}{4 m}\langle\mathrm{x}, \mathrm{x}\rangle} \mathrm{d} \lambda_{E}(\mathrm{x}) \cdot \mathrm{c} \cdot(4 \mathrm{~m})^{-\mathrm{n} / 2} \int_{m}\left(\left.\mathrm{~h}_{A}\right|_{M}\right)^{\wedge} \mathrm{d} \lambda_{m} \\
(4 \mathrm{~m})^{\mathrm{n} / 2} \cdot(4 \mathrm{~m})^{-\mathrm{n} / 2} \cdot \mathrm{c}^{2} \cdot 1=\mathrm{c}^{2},
\end{gathered}
$$

which proves 45 . Q.E.D.

LEMMA 5. Let $S$ be an open subgroup of $G$ and $f$ an element of $L^{1}(G)$ satisfying conditions (40) and (44) of Theorem 3. Then

$$
\check{\mathbf{f}}^{\wedge}=\check{\mathbf{f}}^{\wedge}(0) \cdot \mathrm{f} \text {. }
$$

PROOF. We evidently may assume that $\left.f\right|_{S} \in P_{2}(S, T)$. Let $\&$ be the dual of $S$ and $\sigma \mid \&^{\mathbb{C}} \rightarrow s^{\mathbb{C}}$ the canonical epimorphism as in (46). By (23) of Theorem 1 there exists a function $\mathrm{w} \mid \mathrm{s} \rightarrow g^{\mathbb{C}}$ such that

$$
f_{-x}=f(x) \cdot \overline{w(x)} \cdot f \quad \text { for all } x \in S .
$$

For each $x \in G$, (51), and (60), and (36) yield

$$
\begin{aligned}
& \breve{f}^{\wedge}(x)=\int_{\mathscr{E}} \int_{G} f(t) \quad t \ominus y d \lambda_{G}(t)(-x) \bullet y d \lambda_{\&}(y)= \\
& \int_{\delta} \int_{S} f(t) t \bullet z d \lambda_{G}(t)-x \otimes z d \lambda_{g}(z)=\int_{\delta} \int_{S} f f_{-x}(t) t \bullet z d \lambda_{S}(t) d \lambda_{g}(z)= \\
& f(x) \int_{S} \int_{S} f(t) t \in(z+w(x)) d \lambda g(z)= \\
& f(x) \int_{S} \int_{S} f(t) t \otimes z d \lambda_{S}(t) d \lambda_{g}(z)=f(x) \int_{\mathscr{E}} \int_{G} f(t) \text { toz d } \lambda_{G}(t) d \lambda_{\&}(z)= \\
& f(x) \cdot \breve{f}^{\wedge}(0) \text { Q Q.E.D. }
\end{aligned}
$$


THEOREM 4. (Fourier Inversion and Uniqueness Theorems). There exists a normalization of $\lambda_{\&}$ such that, whenever $h \in L^{1}(G)$ satisfies $\hat{h} \in L^{1}(\&)$, then

$$
\hat{\mathrm{h}}^{2}=\mathrm{h} \quad \lambda_{\mathrm{G}}-\mathrm{a} \cdot \mathrm{e} \text {. }
$$

Further, for any nonzero measure $\mu \in \mathbb{R}(G)$,

$$
\hat{\mu} \neq 0 \text {. }
$$

PROOF. Let $S$ and $a$ be as in Theorem 3 and normalize $\lambda_{\&}$ such that the number in (45) is unity.

Suppose first that $h$ is bounded and continuous. Assume $h(a) \neq \hat{h}^{2}(a)$ for some $a \in G$. Choose $A \in \mathfrak{N}$ such that, if $w \equiv h(a)-\hat{h}^{\vee}(a)$, then

$$
\left|h_{a}(x)-\left(\hat{h}^{2}\right)_{a}(x)-w\right|<\frac{|w|}{2} \text { for all } x \in A \text {. }
$$

By (42) there exist $f \in G$ and $B \in N$ such that $B \subset A$ and

$$
\int_{B} f d \lambda_{G} \geq 2 \frac{\|h\|_{\infty}+\left\|\hat{h}^{`}\right\|_{\infty}}{|w|} \int_{G \backslash B} f d \lambda_{G}
$$

Consequently

$$
\left|\int_{G}\left[h_{a}-\left(\hat{h}^{\nu}\right)_{a}\right] \cdot f d \lambda_{G}\right| \geq \int_{B} \frac{|w|}{2} f d \lambda_{G}-\int_{G \backslash B}\left(\|h\|_{\infty}+\left\|\hat{h}^{\sim}\right\|_{\infty}\right) f d \lambda_{G}>0 .
$$

Direct calculation shows

$$
\left(\hat{g}^{\vee}\right)_{a}=\left(g_{a}\right)^{\wedge} \text { for all } g \in L^{1}(G) \text { with } \hat{g} \in L^{1}(\xi) \text {, }
$$

and application of Fubini's Theorem yields

$$
\int_{G} \not d \quad d \lambda_{G}=\int_{\&} g \ell d \lambda_{\&} \quad \text { for all } g \in L^{1}(\&), \quad \ell \in \mathrm{L}^{1}(G) \text {. }
$$

Then (65), (66) and Lemma 5 yield

$$
\begin{gathered}
\int_{G}\left(\hat{\mathrm{h}}^{\curlyvee}\right)_{\mathrm{a}} \cdot \mathrm{f} \mathrm{d} \lambda_{G}=\int_{G}\left(\mathrm{~h}_{\mathrm{a}}\right)^{\wedge \sim} \cdot \mathrm{f} \mathrm{d} \lambda_{G}=\int_{\&}\left(\mathrm{~h}_{\mathrm{a}}\right)^{\wedge} \hat{\mathrm{f}} \mathrm{d} \lambda_{g}= \\
\int_{G} \mathrm{~h}_{\mathrm{a}} \hat{\mathrm{f}}^{\curlyvee} \mathrm{d} \lambda_{\&}=\int_{G} \mathrm{~h}_{\mathrm{a}} \mathrm{f} \mathrm{d} \lambda_{G}
\end{gathered}
$$

which contradicts (64). Thus $h=\hat{h}^{\curlyvee}$.

If $\mu \in \mathbb{P}(G)$ is nonzero, there exists a continuous function $\mathrm{g} \mid \mathrm{G} \rightarrow \mathrm{C}$ with compact support such that $\int_{G} g(-t) d \mu(t) \neq 0$. Then the continuous function $g * \mu$ is nonzero at 0 and we may define

$A \equiv\left\{x \in S:|g * \mu(-x)|>\frac{|g * \mu(0)|}{2}\right.$. By (42) there exist $f \in G$ and $B \in \mathbb{R}$ 
such that $B \subset A$ and

$$
\int_{B} f d \lambda_{G}>\frac{2\left\|_{g} * \mu\right\|_{\infty}}{|g * \mu(0)|} \quad \int_{G} \bigwedge_{B} f d \lambda_{G} .
$$

Thus

$$
\left|\int_{G} g * \mu(t) \cdot f(t) d \lambda_{G}(t)\right|>\int_{B} \frac{|g * \mu(0)|}{2} f d \lambda_{G}-\int_{G \backslash B}\|g * \mu\|_{\infty} \cdot f d \lambda_{G}>0 .
$$

Hence $f * g * \mu$ is nonzero continuous and

$$
\|(\mathbf{f} * g * \mu) \hat{\imath}\|_{1}=\|\hat{f} \cdot(g * \mu) \hat{n}\|_{1} \leq\|\hat{\mathbf{f}}\|_{1} \cdot\|(g * \mu) \hat{\wedge}\|_{\infty}<\infty .
$$

Thus (61) implies that $(f * g * \mu)^{\wedge}=f * g * \mu$. Hence $0 \neq(f * g * \mu)^{\wedge}=(f * g)^{\wedge} \cdot \hat{\mu}$, which establishes (62).

Now suppose that $h \in L^{1}(G)$ satisfying $\hat{h} \in L^{1}(\&)$ is arbitrary. Let $f_{\alpha}$ be the net given by (43) of Theorem 3 and, for each index $\alpha$, let $g_{\alpha} \equiv \mathrm{f}_{\alpha} /\left\|\mathrm{f}_{\alpha}\right\|_{1}$.

Then $\lim _{\alpha}\left\|\hat{\mathrm{h}}-\hat{\mathrm{g}}_{\alpha} \cdot \hat{\mathrm{h}}\right\|_{1}=0$ so

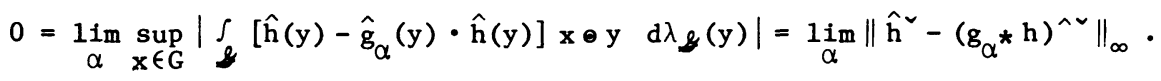

Because each $g_{\alpha} * h$ is continuous and $\left\|\left(g_{\alpha} * h\right)^{\wedge}\right\|_{1} \leq\|\hat{g}\|_{\infty}\|\hat{h}\|_{1}<\infty$, it follows from (61) that $\left(g_{\alpha} * h\right)^{\wedge \sim}=g_{\alpha} * h$. If $\hat{h}^{\sim}$ were not in $L^{1}(G)$, there would exist $\ell \in L^{1}(G) \cap L^{\infty}(G)$ such that

$$
\left.\int_{G} \hat{\mathrm{h}}\right\urcorner \ell d \lambda_{G}>\|\mathbf{h}\|_{1} \cdot\|\ell\|_{\infty} ;
$$

but (67) implies

$$
\left|\int_{G} \hat{h}^{\sim} \ell \mathrm{d} \lambda_{G}\right|=\underset{\alpha}{\operatorname{1im}}\left|\int_{G} g_{\alpha} * h \cdot \ell d \lambda_{G}\right| \leq \overline{\lim }\left\|g_{\alpha}\right\|_{1} \cdot\|\mathrm{h}\|_{1} \cdot\|\ell\|_{\infty}=\|\mathrm{h}\|_{1}\|\ell\|_{\infty} ;
$$

an absurdity. Thus, $\hat{h}^{\vee} \in L^{1}(G)$. Interchanging the roles of $\wedge$ and $\star$, and of $G$ and \&, the continuity of $\hat{h}$ and (61) imply that $\hat{h}^{\sim \wedge}=\hat{h}$. It now follows from (62) that $\hat{h}^{`}=h$. Q.E.D. 


\section{REFERENCES}

1. GUICHARDET, A. Analyse Harmonique Commutative. Dunod, Paris, 1968.

2. HEWITT, Edwin and Ross, Kenneth, Abstract Harmonic Analysis, Vol. I, Academic Press, New York; Springer-Verlag, Ber1in, 1963.

3. , Abstract Harmonic Analysis. Vol. II, Springer-Verlag, New York and Berlin, 1970.

4. NOVAK, David and McKennon, Kelly, Exponentials on Locally Compact Abelian Groups, Proc. Am. Math. Soc., 83 (1981), 307-314.

5. RAIKOV, D.A. Positive Definite Functions on Commutative Groups with an Invariant Measure, Dok1. Aka. Mank SSSR, N.S. 28 (1940), 296-300.

6. RUDIN, Walter, Fourier Analysis on Groups. Interscience Publishers, New York and London, 1962.

7. VAN KAMPEN, E.R. Locally Bicompact Abelian Groups and their Character Groups, Ann. of Math. (2) 36 (1935), 448-463. 


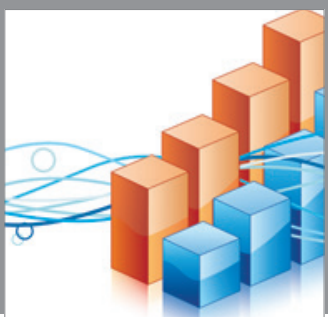

Advances in

Operations Research

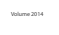

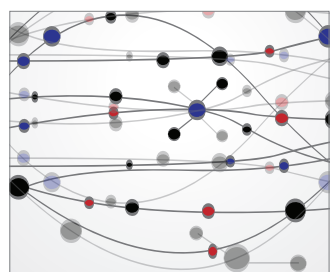

\section{The Scientific} World Journal
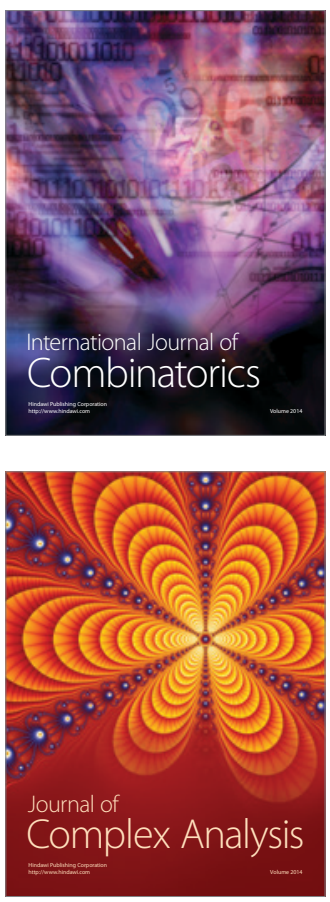

International Journal of

Mathematics and

Mathematical

Sciences
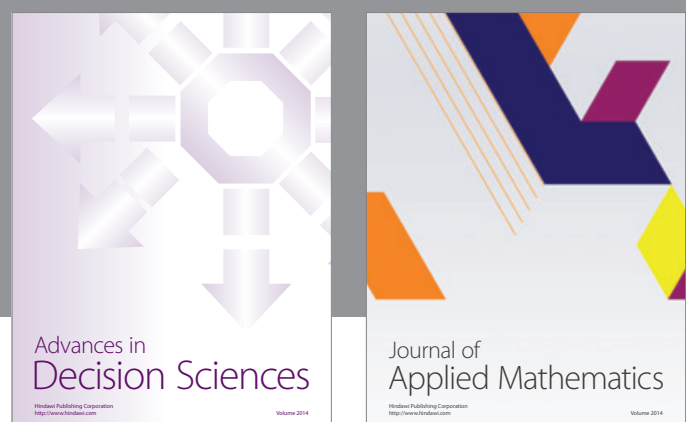

Journal of

Applied Mathematics
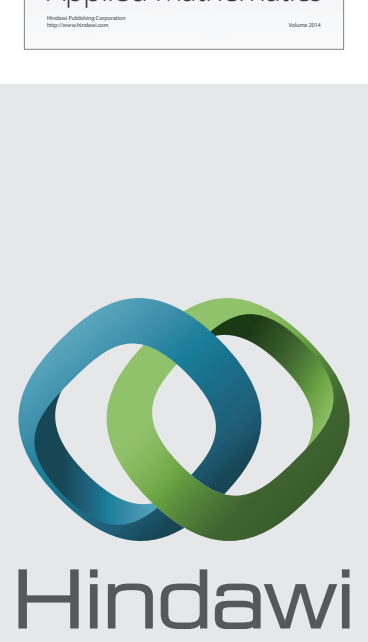

Submit your manuscripts at http://www.hindawi.com
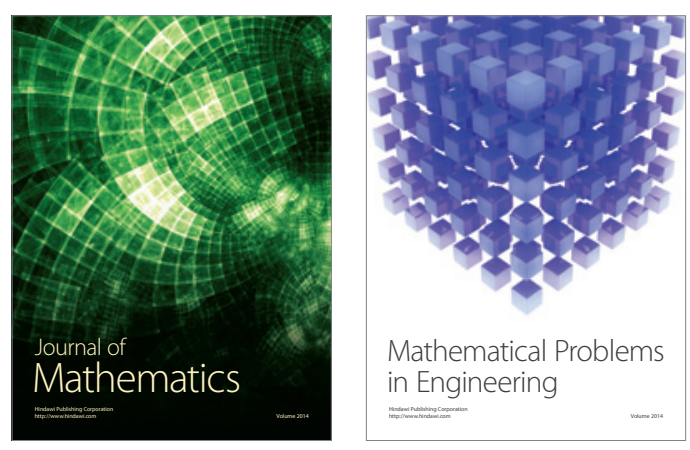

Mathematical Problems in Engineering
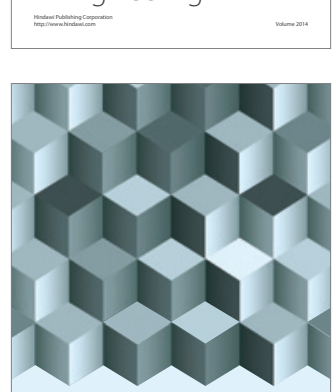

Journal of

Function Spaces
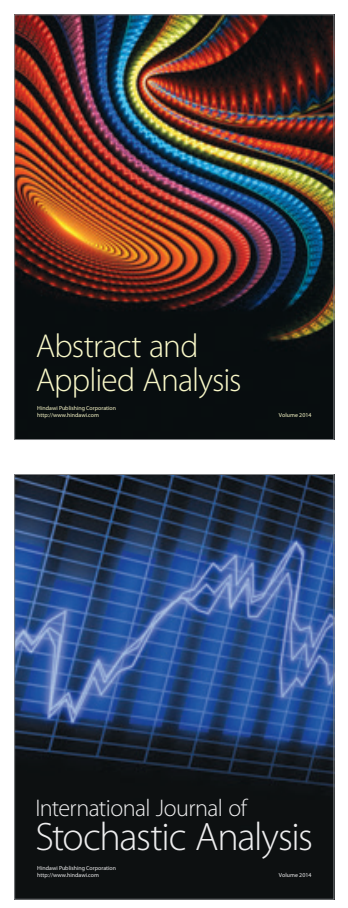

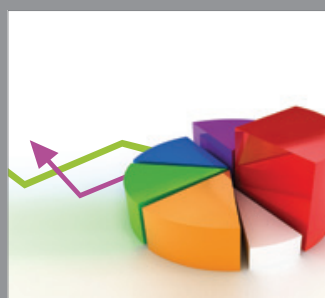

ournal of

Probability and Statistics

Promensencen
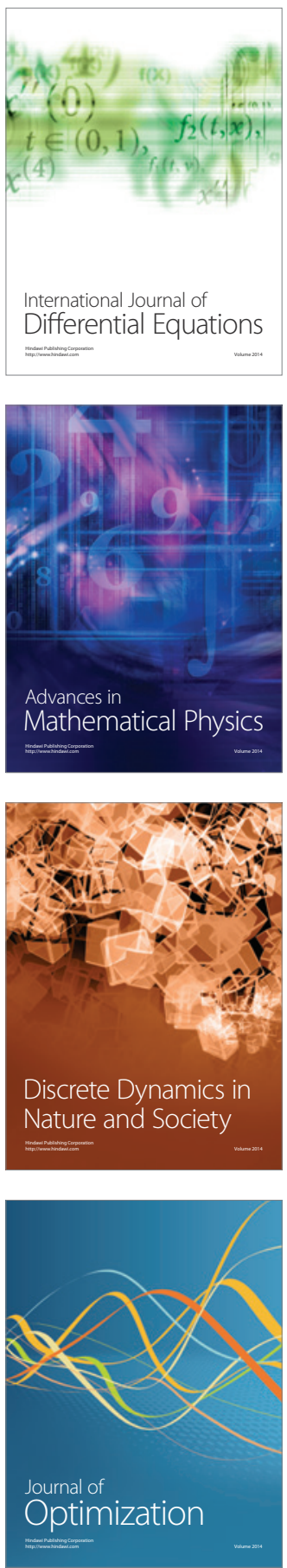